\title{
Optimum quantum states for interferometers with fixed and moving mirrors
}

\author{
Alfredo Luis* \\ Departamento de Óptica, Facultad de Ciencias Físicas, Universidad Complutense, 28040 Madrid, Spain
}

(Received 9 November 2003; published 22 April 2004)

\begin{abstract}
We address a systematic approach to the study of the optimum states reaching maximum resolution for interferometers with moving mirrors. We find a correspondence between the optimum states for interferometers with fixed and moving mirrors.
\end{abstract}

DOI: 10.1103/PhysRevA.69.045801

PACS number(s): 42.50.St, 03.65.-w

Interferometric measurements are among the most sensitive measuring strategies currently available. This has prompted a significant amount of effort devoted to the investigation of the limits that quantum fluctuations may impose on the precision of interferometric measurements. This means determining two items: the maximum resolution and the optimum input states required to achieve it.

A well studied example is the case of the interferometers with moving mirrors, devised to detect minute forces acting on them, such as the pass of a gravitational wave, for example, Ref. [1]. While the issue of maximum resolution has been well studied [2-8], much less effort has been devoted to the topic of the optimum states. Leaving aside a very recent work [9], only quadrature coherent and squeezed states have been considered [2-8]. This situation is in sharp contrast to the case of standard interferometry with fixed mirrors, where many different families of states have been proposed in order to reach the quantum limits [10-13].

In this work we address a systematic approach to the study of the states reaching maximum resolution for interferometers with moving mirrors. We solve the problem by finding a one-to-one correspondence between the optimum states for interferometers with fixed and moving mirrors. The key point to this result is a fully quantum description of the interferometer as a nonlinear input-output transformation embodying the mobility of the mirrors [5-8]. This approach has been already useful to demonstrate that the precision can be arbitrarily increased by increasing the power of the input light, as demonstrated in Refs. $[4,6,8]$, in sharp contrast to the previous idea of an upper bound independent of the intensity (the standard quantum limit) $[2,3,5,7]$. In this work we show that the limits to the resolution of interferometers with moving and fixed mirrors are the same. We also show that the input-output approach allows us to easily solve the question of the optimum states.

For definiteness we focus on the Michelson interferometer schematized in Fig. 1 showing the input $a_{1,2}$, internal $b_{1,2}$, $\tilde{b}_{1,2}$, and output $\tilde{a}_{1,2}$ modes whose complex amplitude operators are coupled at the beam splitter:

$$
b_{1}=\frac{1}{\sqrt{2}}\left(a_{1}+a_{2}\right), \quad \tilde{a}_{1}=\frac{1}{\sqrt{2}}\left(\tilde{b}_{1}+\tilde{b}_{2}\right),
$$

\footnotetext{
*Electronic address: alluis@ fis.ucm.es
}

$$
b_{2}=\frac{1}{\sqrt{2}}\left(a_{1}-a_{2}\right), \quad \tilde{a}_{2}=\frac{1}{\sqrt{2}}\left(\tilde{b}_{1}-\tilde{b}_{2}\right) \text {. }
$$

Taking into account the mobility of the mirrors the inputoutput transformation can be expressed as

$$
\tilde{a}_{j}=U_{\mathrm{M}}^{\dagger} a_{j} U_{\mathrm{M}}, \quad \tilde{b}_{j}=U_{\mathrm{M}}^{\dagger} b_{j} U_{\mathrm{M}},
$$

where

$$
U_{\mathrm{M}}=U_{\mathrm{F}} U_{\mathrm{P}}=U_{\mathrm{P}} U_{\mathrm{F}}
$$

and

$$
\begin{gathered}
U_{\mathrm{F}}=e^{i 2 k\left(L_{1} b_{1}^{\dagger} b_{1}+L_{2} b_{2}^{\dagger} b_{2}\right)}, \\
U_{\mathrm{P}}=e^{i 2 g\left[\left(b_{1}^{\dagger} b_{1}\right)^{2}+\left(b_{2}^{\dagger} b_{2}\right)^{2}\right]},
\end{gathered}
$$

$L_{1}, L_{2}$ being the lengths of the arms in darkness, $k$ the wave number, and $g$ a constant depending on the mass and restoring forces of the mirrors, assumed identical [3-8]. The signal to be detected would produce a change of the phase difference $\phi=2 k\left(L_{2}-L_{1}\right)$ which in turn is observed as a variation of the output intensities.

In the above expressions $U_{\mathrm{F}}$ represents the input-output transformation for the same interferometer but with fixed mirrors, while the nonlinear term $U_{\mathrm{P}}$ accounts for the effect of the radiation pressure. It is worth noting that both factors commute $\left[U_{\mathrm{F}}, U_{\mathrm{P}}\right]=0$. This is the key point that allows us to establish the equivalence between interferometers with fixed

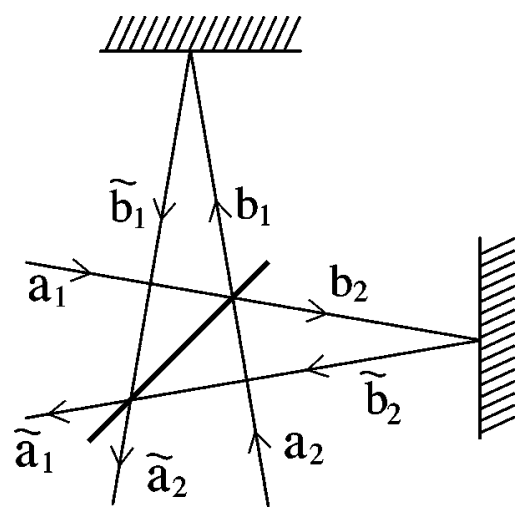

FIG. 1. Scheme of a Michelson interferometer showing the input, output, and internal modes. For the sake of illustration the incidence has been represented as non-normal. 
and moving mirrors. To this end let us express the input state $|\psi\rangle_{\mathrm{M}}$ for interferometer with moving mirrors as

$$
|\psi\rangle_{\mathrm{M}}=U_{\mathrm{P}}^{\dagger}|\psi\rangle_{\mathrm{F}}
$$

so that the output state is

$$
|\tilde{\psi}\rangle_{\mathrm{M}}=U_{\mathrm{M}}|\psi\rangle_{\mathrm{M}}=U_{\mathrm{F}}|\psi\rangle_{\mathrm{F}}
$$

This is the same output state for an interferometer with fixed mirrors illuminated by the input state $|\psi\rangle_{\mathrm{F}}$. Therefore, if $|\psi\rangle_{\mathrm{F}}$ is optimum for fixed mirrors, then $|\psi\rangle_{\mathrm{M}}$ is optimum for moving mirrors, irrespective of the measurement performed at the output fields. This implies two conclusions: (i) the limits to the resolution of interferometers with moving and fixed mirrors are the same and (ii) the corresponding optimum states are related by Eq. (5).

Concerning the first conclusion, it is known that for interferometers with fixed mirrors the minimum phase uncertainty scales as the inverse of the mean number of photons employed in the measurement (this is referred to as the Heisenberg limit) $[10,13]$. From the first result listed above we get that this is also the case of interferometry with moving mirrors. This is in sharp contrast with the idea of a lower limit independent of the number of photons (the standard quantum limit) $[2,3,5,7]$. Previous works on the subject have already demonstrated that the standard quantum limit may be surpassed by a proper choice of the input states $[4,6,8,14]$. In this work we have demonstrated in very general terms that any improvement of the precision beyond the standard quantum limit is actually bounded by the Heisenberg limit, as being the ultimate quantum limit for interferometry with moving mirrors. The second conclusion above greatly increases the possibilities to reach the quantum limits by using very diverse input states and measuring strategies that have already been studied in the context of interferometry with fixed mirrors, involving input states such as SU(2) squeezed states or number states [10-12]. As an application illustrating the results of this work this last example will be examined below in some detail.

We can regard this last result from two different perspectives. On the one hand we can consider that the interferometer is actually illuminated by the state (5). On the other hand, Eq. (6) suggests that the optimization consists essentially of the removal of the radiation pressure while the interferometer is illuminated directly by the input state $|\psi\rangle_{\mathrm{F}}$. This idea of compensation of radiation pressure has been studied in Refs. [3,4]. In any case, the transformation $U_{\mathrm{P}}^{\dagger}$ can be accomplished in practice by propagation in nonlinear Kerr medium.

The two conclusions above are direct consequences of a fully quantum description of the interferometer as a nonlinear input-output transformation embodying the mobility of the mirrors as introduced and developed in Refs. [5-8]. The uniqueness of the present paper with respect to the previous works are the three following results. We have used the input-output approach to derive a complete equivalence between interferometers with fixed and moving mirrors. We have found that the ultimate quantum limit to the sensitivity of interferometry with moving mirrors is the Heisenberg limit. We have found the quantum states reaching this limit for different measuring strategies previously introduced within the context of interferometry with fixed mirrors.

Next we illustrate these points with a suitable particular example. We consider as input state $|\psi\rangle_{\mathrm{F}}=|n\rangle_{1}|n\rangle_{2}$ a product of an equal number of photons in the input modes $a_{1,2}$ [12]. For the sake of completeness we include the possibility that the transformation $U_{\mathrm{P}}$ in Eq. (5) is slightly different from $U_{\mathrm{P}}$ in Eq. (3), so that the removal of the radiation pressure is not complete. This can be taken into account by considering that $|n\rangle_{1}|n\rangle_{2}$ is the input state for the transformation $U_{\mathrm{M}}$ after replacing the parameter $g$ by a new parameter $\chi$ which would be zero in the case of perfect compensation of the radiation pressure.

Since the input state is eigenvector of the total number operator we find it helpful to rearrange $U_{\mathrm{P}}$ in the form

$$
\left(b_{1}^{\dagger} b_{1}\right)^{2}+\left(b_{2}^{\dagger} b_{2}\right)^{2}=\frac{1}{2}\left(S_{0}^{2}+S_{x}^{2}\right),
$$

where $S_{j}, j=0, x, y, z$ denote the Stokes operators

$$
\begin{gathered}
S_{0}=b_{1}^{\dagger} b_{1}+b_{2}^{\dagger} b_{2}=a_{1}^{\dagger} a_{1}+a_{2}^{\dagger} a_{2}, \\
S_{x}=b_{1}^{\dagger} b_{1}-b_{2}^{\dagger} b_{2}=a_{1}^{\dagger} a_{2}+a_{2}^{\dagger} a_{1}, \\
S_{y}=i\left(b_{1}^{\dagger} b_{2}-b_{2}^{\dagger} b_{1}\right)=i\left(a_{2}^{\dagger} a_{1}-a_{1}^{\dagger} a_{2}\right), \\
S_{z}=b_{1}^{\dagger} b_{2}+b_{2}^{\dagger} b_{1}=a_{1}^{\dagger} a_{1}-a_{2}^{\dagger} a_{2} .
\end{gathered}
$$

Performing a similar rearrangement for the linear part $U_{\mathrm{F}}$ we get that for this input state the total input-output transformation can be expressed as

$$
U_{\mathrm{M}}=e^{i(\phi / 2) S_{x}} e^{i \chi S_{x}^{2}} .
$$

Since for the output state $\langle\boldsymbol{S}\rangle=0$ the simple measurement of the output intensities does not provide suitable phasedependent observables and other strategies must be adopted [12]. For example, we can consider $M=S_{z}^{2}$ as the measured observable at the output. A suitable estimator for the phase uncertainty is

$$
\Delta \phi=\frac{\langle\Delta M\rangle}{|d\langle M\rangle / d \phi|} .
$$

For $\chi=0$ (perfect removal of the radiation pressure) we get

$$
\begin{gathered}
\langle M\rangle \simeq 2 n^{2} \sin ^{2} \phi, \\
\left\langle(\Delta M)^{2}\right\rangle \simeq 2 n^{4} \sin ^{2} \phi+2 n^{2} \sin ^{2}(2 \phi),
\end{gathered}
$$

so that

$$
(\Delta \phi)^{2} \simeq \frac{\tan ^{2} \phi}{8}+\frac{1}{2 n^{2}}
$$

and the maximum resolution (Heisenberg limit) is obtained for $\phi=0, \pi$. 
On the other hand, for $\chi \neq 0$ (partial removal of the radiation pressure) and for high enough $n$ we can use in Eq. (9) the approximation $S_{x} \simeq 2 n \cos \theta$, where $\theta$ is defined by the ladder operators (for $n>1$ )

$$
e^{ \pm i \theta}|n\rangle_{1}|n\rangle_{2}=|n \pm 1\rangle_{1}|n \mp 1\rangle_{2} .
$$

For $n \gg 1$ these operators can be treated as effectively unitary $e^{ \pm i \theta} e^{\mp i \theta} \simeq e^{\mp i \theta} e^{ \pm i \theta} \simeq 1$. Since the radiation pressure is a small effect, and for preparations of the interferometer around the above optimum working point $\phi=0$, we can expand the result in powers of $\phi, \chi$ retaining up to second order

$$
\begin{gathered}
\langle M\rangle \simeq 2 \phi^{2} n^{2}+32 \chi^{2} n^{4}+\cdots, \\
\langle(\Delta M)\rangle^{2} \simeq 8 \phi^{2} n^{2}+512 \chi^{2} n^{4}+\cdots,
\end{gathered}
$$

and

$$
(\Delta \phi)^{2} \simeq \frac{1}{2 n^{2}}+\frac{32 \chi^{2}}{\phi^{2}} .
$$

In this case the optimum resolution requires that $\chi \ll \phi$. Otherwise very large phase fluctuations would arise. In any case, when $n \rightarrow \infty$, the phase uncertainty attains a lower bound $\Delta \phi=4 \sqrt{2} \chi / \phi$ that no longer depends on the number of photons employed. Therefore, the input number state is extremely sensitive to errors and imperfections in the compensation of the radiation pressure.

It is worth extracting some further conclusions from the above calculations. For interferometers with moving mirrors there are two basic sources of quantum uncertainty: the shot noise and the fluctuations of the radiation pressure. Two major approaches have been followed to compute the joint effect of these sources. On the one hand, there is a separate calculation that considers the noise sources as if they were statistically independent, leading to the standard quantum limit $[2,9]$. On the other hand, in a unified calculation (the approach followed in this work) the mobility of the mirrors is embodied in the input-output transformation, so that all the uncertainties manifest as shot noise [3-8]. In comparison with the separate calculation, the results of this work provide two points of discrepancy.
In a separate calculation the shot noise is computed as if there were no radiation pressure. Since this last one does not depend on $\phi$, the separate analysis of this particular strategy followed in Ref. [9] concludes that $\phi=0$ is the optimum working point with and without radiation pressure. On the other hand, we have shown above that according to the unified calculation the optimum value for $\phi$ is no longer $\phi=0$. As a matter of fact, $\phi=0$ would provide very bad results when $\chi \neq 0$. We think that the unified approach provides the correct result. This is because around $\phi=0$ we get $d\langle M\rangle / d \phi \simeq 0$ and the small uncertainty in $M$ caused by the fluctuations of the radiation pressure must imply a very large phase uncertainty $\Delta \phi$.

We can also appreciate that the dependence of the contribution of radiation pressure with the input intensity [the last terms in Eqs. (14)] differs from the result of a separate calculation [9]. This is because in the unified calculation the radiation pressure effects are seen through the fluctuations of the square of $S_{z}$. This modifies the dependence with the intensity in comparison with a direct calculation, where the effect of radiation pressure is computed irrespective of the detection mechanism. It is worth stressing that this refers to the comparison between separate and unified calculations just for a particular measuring arrangement (input number states and detection of the squared difference of output photon numbers) so that the conclusion would not apply to other different strategies such as the one considered in Ref. [2], for example.

Summarizing, we have presented and completed a systematic approach to the optimization of interferometers with moving mirrors by finding an equivalence with the same problem for fixed mirrors. This allows us to translate to the former all the results already proved for the latter, including the determination of the optimal input states. For the demonstration of the above results it is crucial to describe the interferometer with moving mirrors by a fully quantum nonlinear input-output transformation embodying the mobility of the mirrors. Finally, the particular example considered above reveals the advantages of the input-output formalism including radiation pressure in comparison with other approaches.

This work has been supported by Project No. PR1/0311630 of the University Complutense.
[1] E. D. Black and R. N. Gutenkunst, Am. J. Phys. 71, 365 (2003).

[2] C. M. Caves, Phys. Rev. Lett. 45, 75 (1980); Phys. Rev. D 23, 1693 (1981).

[3] R. S. Bondurant, Phys. Rev. A 34, 3927 (1986).

[4] A. F. Pace, M. J. Collett, and D. F. Walls, Phys. Rev. A 47, 3173 (1993).

[5] R. Loudon, Phys. Rev. Lett. 47, 815 (1981).

[6] W.-T. Ni, Phys. Rev. D 35, 3002 (1987); O. Assaf and Y. Ben-Aryeh, J. Opt. B: Quantum Semiclassical Opt. 4, 49 (2002); J. Opt. Soc. Am. B 19, 2716 (2002).

[7] A. Luis and L. L. Sánchez-Soto, J. Mod. Opt. 38, 971 (1991).

[8] A. Luis and L. L. Sánchez-Soto, Opt. Commun. 89, 140
(1992); Phys. Rev. A 45, 8228 (1992).

[9] C. Brif, Phys. Lett. A 263, 15 (1999).

[10] A. Luis and L. L. Sánchez-Soto, in Progress in Optics, edited by E. Wolf (Elsevier, Amsterdam, 2000), Vol. 41, p. 421 and references therein.

[11] B. Yurke, Phys. Rev. Lett. 56, 1515 (1986); B. Yurke, S. L. McCall, and J. R. Klauder, Phys. Rev. A 33, 4033 (1986); M. Kitagawa and M. Ueda, Phys. Rev. Lett. 67, 1852 (1991); Phys. Rev. A 47, 5138 (1993); M. Hillery and L. Mlodinow, ibid. 48, 1548 (1993); G. S. Agarwal and R. R. Puri, ibid. 49, 4968 (1994); A. Bandyopadhyay and J. Rai, ibid. 51, 1597 (1995); C. Brif and A. Mann, ibid. 54, 4505 (1996); J. J. Bollinger, W. M. Itano, D. J. Wineland, and D. J. Heinzen, 
ibid. 54, R4649 (1996); Z. Y. Ou, ibid. 55, 2598 (1997); J. P. Dowling, ibid. 57, 4736 (1998); C. C. Gerry, ibid. 61, 043811 (2000); C. C. Gerry and A. Benmoussa, ibid. 65, 033822 (2002); W. J. Munro, K. Nemoto, G. J. Milburn, and S. L. Braunstein, ibid. 66, 023819 (2002); C. P. Search and P. Meystre, ibid. 67, 061601(R) (2003); A. G. Rojo, ibid. 68, 013807 (2003); C. C. Gerry and R. A. Campos, ibid. 68, 025602 (2003).

[12] M. J. Holland and K. Burnett, Phys. Rev. Lett. 71, 1355 (1993); M. Hillery, M. Zou, and V. Bužek, Quantum Semiclassic. Opt. 8, 1041 (1996); P. Bouyer and M. Kasevich, Phys. Rev. A 56, R1083 (1997); T. Kim, O. Pfister, M. J. Holland, J.
Noh, and J. L. Hall, ibid. 57, 4004 (1998); T. Kim, O. Pfister, M. J. Holland, J. Noh, and J. L. Hall, ibid. 58, 2617 (1998); T. Kim, J. Shin, Y. Ha, H. Kim, G. Park, T. G. Noh, and C. K. Hong, Opt. Commun. 156, 37 (1998); T. Kim, Y. Ha, J. Shin, H. Kim, G. Park, K. Kim, T.-G. Noh, and Ch. K. Hong, Phys. Rev. A 60, 708 (1999); V. Meyer, M. A. Rowe, D. Kielpinski, C. A. Sackett, W. M. Itano, C. Monroe, and D. J. Wineland, Phys. Rev. Lett. 86, 5870 (2001); A. Dunningham, K. Burnett, and S. M. Barnett, ibid. 89, 150401 (2002).

[13] Z. Y. Ou, Phys. Rev. A 55, 2598 (1997).

[14] M. T. Jaekel and S. Reynaud, Europhys. Lett. 13, 301 (1990). 